\title{
Agility in Project Management Phases by Scrum Method
}

\author{
Sara Mahmoudi * \\ Department of computer \\ enginering, University of \\ Science and Technology, \\ Babol, Mazandaran, Iran
}

\author{
Hooman Kashanian \\ Department of computer \\ enginering ,Islamic Azad \\ University, Ferdows, \\ Khorasan, Iran
}

\author{
Mehdi Ghasemi Farsad \\ Department of computer \\ enginering ,Islamic Azad \\ University, Hamedan , \\ Hamedan, Iran
}

\begin{abstract}
Agile methodology is a set of methods that cause the produced software to adjust with customers' needs completely. For combination of agile methodology and project management we sugestion one methodology, This methodology is designed to meet the needs of all segments of the organization as they engage in technical project work. It serves as a guide to the organization as it selects its projects, to project teams as they plan the work, to management as they supply the required oversight, and to Sponsors and Clients as they collaborate in the design and delivery of new business systems. In fact, the customer is fully associated with the project team. Current perceptions and emerging trends of various Software project management practices are reviewed and risks specific to software development projects are identified. Implementing effective project management process will succeed by changing the organizational culture. One method of agile is scrum in which the development team meet the customer at different intervals and deliver them an output of the software and see feedback. Traditional modeling methods are based on documentations and stationary reports which are not responsible for increasing changes of users' needs in environments that are changing rapidly. Pre-development heavy designing and primary comprehensive and majorplanning in traditional methods made experts to think about new methods to develop software Keywords: Agile, methodology, project management, scrum
\end{abstract}

\section{INTRODUCTION}

Software development and agile project management has been rapidly gaining popularity in the software industry over the past two decades (Dingsøyr, Nerur, Balijepally \& Ministry of Education; Begel \& Nagappanand,2007).

Agile software development is a conceptual framework for undertaking software engineering projects. There are a number of agile software development methodologies e.g. Crystal Methods, Dynamic Systems Development Model (DSDM), and Scrum Most agile methods attempt to minimize risk by developing software in short timeboxes, called iterations, which typically last one to four weeks. Each iteration is like a miniature software project of its own, and includes all the tasks necessary to release the mini-increment of new functionality: planning, requirements analysis, design, coding, testing, and documentation. While an iteration may not add enough functionality to warrant releasing the product, an agile software project intends to be capable of releasing new software at the end of every iteration. At the end of each iteration, the team reevaluates project priorities.

Considering ever growing of internet users all around the world, software demands are expanding as well. Due to technologies changes and innovations in operating systems, the demands of users for creating and updating the software are changing in an uncontrolled manner[10].

Agile methods emphasize realtime communication, preferably face-to-face, over written documents. Most agile teams are located in a bullpen and include all the people necessary to finish the software. At a minimum, this includes programmers and the people who define the product such as product managers, business analysts, or actual customers. The bullpen may also include testers, interface designers, technical writers, and management.

\section{PROJECT AND PROJECT MANAGEMENT}

A project is defined as "a temporary endeavor undertaken to create a unique product or service" (PMI, 2000). A project is undertaken when work is best accomplished through

methods that fundamentally differ from those of everyday operations. A list of the key

characteristics of a project can further clarify that definition:

- Temporary endeavor with a beginning and an end

- Often broken into subprojects (or phases)

- Creates a unique product or service

- Done for a purpose

- Has interrelated activities (tasks)

- Is an instrument of change

A project usually has certain aspects or key components which include project-related

management, a common vocabulary, project-related methods and tools, teamwork, a plan,

trade-offs (involving scope/deliverables, time, cost, and quality), identified requirements

(needs) and unidentified requirements (wants or expectations), and stakeholders.

The stakeholders involved with a project may be many and possibly diverse in several

respects including interests, needs, expectations, and priorities. Satisfying the stakeholders

is one of the key objectives of the project and the project manager. Key

stakeholders include the organization and people doing the work, who are called the

"performing organization," and the people or organization benefiting from the work (and

also usually paying for the work), who are called the "benefiting organization." These 
two organizations may or may not belong to the same corporation. In below we define project management phases for agility [9].

\subsection{PHASE1-PROJECT INITIATION}

Projects may come about for a variety of reasons and they may present themselves at any time. The selection process is carried out during Initiation. The Initiation Process is that time in the lifecycle of a project when the project idea is defined, evaluated, and authorized. The executive committee at UNCG is our Project Review Committee (PRC) that meets on a weekly basis. Each division at the University creates a ranked list of projects. During the PRC meetings, the highest ranked projects in each division are selected for "sizing \& scoping" effort to determine which resources are needed and if the project is feasible given available resources. This process gives management and other stakeholders an opportunity to validate the project's potential benefits realization [8].

\subsection{PHASE2 - PROJECT PLANNING}

Project Planning follows the Project Initiation phase and is considered to be the most important stage in project management. Project Planning is not a single activity or task. It is a process that takes time and attention. Project Planning defines the project activities and describes how the activities will be accomplished. Time spent up-front identifying the proper needs and structure for organizing and managing projects saves countless hours of confusion and rework in the Managing (Execution and Controlling) phase of the project.

\subsection{PHASE3-PROJECT MANAGING}

Once a project moves into the Execution \& Controlling phase, the project manager's main focus during this phase shifts to monitoring the work being done. Managing the project plan ensures that planned project activities are carried out in an effective and efficient manner. A missed activity finish date may require adjustments to the entire project schedule, resource staffing, and other impacts [5].

\subsubsection{Manage Scope}

Scope control is a straightforward concept. The intent of implementing a scope control process is to identify and manage all elements (e.g., people and requirements) inside and outside of the project that increase or decrease the project scope beyond the required or defined need of the original, agreed-upon project Scope Statement.

Scope changes will come from the perceived need for a change in a project deliverable that may affect its functionality and in most cases the amount of work needed to perform the project. A scope change is a very crucial occurrence.

A scope change could require a change in resources time, and/or project funding. All scope change requests should be submitted in writing using the change control process and form. The Project Manager will review the change with the project sponsor and other major stakeholders to determine that the change is necessary and the additional resources are available. Any changes that are agreed upon must be approved by the project sponsor, technical lead, and/or clients as a matter of formal scope control. This can be an email or a change of scope document. A change of scope may cause changes to be made to other project documents such as the schedule/task list and budget. All changes must be communicated to the project team and stakeholders.

\subsubsection{Manage Schedule}

Schedule control is one of the most important activities within project control. It is important for the Project Team to know where the project stands with respect to project schedule (i.e., Is the project ahead of, or behind, schedule, or what tasks do I need to complete by what date?). It becomes key for Project managers to obtain statuses from the team members on a regular basis.

As part of the status collection, the Project Manager should:

Validate that task start and end dates are still accurately reflected.

Validate that task dependencies (or relationships) are still valid.

Validate work effort (or task duration) is still valid in the schedule. If this changes, obtain accurate start and finish dates of completed tasks or estimates to complete work for ongoing tasks.

Schedule control is something that typically is managed at the project level by the Project Manager. However, it is very important to make the client aware that a schedule change has occurred. Furthermore, the client needs to be made aware of what is being done to fix the issue and the impact it will have on the project's performance and deliverables. It is a good practice for Project Managers to hold regular project schedule reviews [7].

It is standard practice to baseline the schedule at the start of the project. This allows all schedule changes to be displayed against the original project schedule. If schedule slippage becomes severe it may be advisable to re-baseline the project. As this involved change to one of the project baselines, it should only be done through a formal Change Control Process.

\subsubsection{Manage Issues}

The Issue Management process should give everyone involved with, or affected by, the project a way to report issues or problems. The Issues Log format provides fields for documenting the problem, assessing the impact of the problem, making recommendations and determining the cost (people and assets) and time required for resolving the problem.

Any of the Project Team members, customers, or Stakeholders can submit an issue. This will be recorded on an Issue log or meeting minutes. All issues should be reviewed on a regular basis (e.g., in the project status meetings, since this group will typically meet on a weekly or biweekly basis).

Typically, when the issue or problem has been resolved and verified, recording the actual date the problem was resolved and the approval authority closes the issue. Some issues may need executive management approval. The appropriate processes will be followed to update contracts and baseline documents. 


\subsubsection{Manage Communications}

The project Communications Plan is an important factor in the Managing phase. A large part of a Project Manager's responsibility during this stage of the project is keeping the Stakeholders informed of project status. There are many facets to project communications. Some examples follow:

Generate status reports to the team and perhaps to executives on a regular basis.

Meeting minutes should be made available to Stakeholders along with any "to-do" lists that may have been generated during the meetings.

The project schedule and other project documentation should be available to the Stakeholders.

Hold regular status meetings.

In addition to these formal communications, a Project Manager should also stay in communication with the team on an informal basis. Informal discussion is sometimes the best way to determine team morale, true project status, looming difficulties, etc.

\subsubsection{Manage Cost}

Projects may fail to control costs, or go over budget, for many reasons. Often it is not a single problem but a series of small problems that, combined, permit cost control to be sacrificed and prevent the project from being completed successfully. Project Managers should monitor the costs as outlined in the Procurement Plan. If costs increase, the PM should search out the "why" and take appropriate action and inform the stakeholders of the authorized changes. Cost control is not simply a reporting process.[4]

UNCG and each of its departments will have a defined set of guidelines and policies that provide the infrastructure for project purchasing that should be integrated within the Procurement Plan. These guidelines will outline the policy for solicitation, source selection and contract administration. Although the solicitation and contracting responsibilities may not always be managed by the Project Manager, it is still important that the Project Manager have a fundamental understanding of the department's contracting and procurement policies.

The Project Manager may be responsible for ensuring that the vendors, once contracted to do the work, meet the contractual agreements specified within their contracts. Project Managers will also be responsible for tracking, reviewing and analyzing the performance of contractors on a project. This performance reporting will be the basis for any contractual changes that need to be made during the life of the contract. Finally, Project Managers may play an important role in oversight and review of any contract changes that will affect the project [3].

\subsubsection{Manage Risk}

Risk identification, monitoring and resolution are important tools for successfully completing a project. Larger projects may need a Risk Log, documenting known risks and any mitigation for those risks. One type of risk on IT projects is the development and implementation of technology equipment and software that might become obsolete very quickly. Technology is evolving rapidly with increases in speed and capabilities. Accordingly, risk is increased when implementing high dollar or homegrown technology systems.
To alleviate this issue, the Project Manager must make sure that the efforts of the Project Team are aligned with the technology and business strategy of the department. Researching future needs, capabilities, and integration requirements of the products will be helpful [5].

\subsubsection{Manage Quality}

Quality assurance incorporates a process of evaluating overall project performance on a regular basis to provide confidence that the project will satisfy the relevant quality standards. Quality control should be performed throughout the project. Project results include both product results, such as deliverables, and management results, such as cost and schedule performance. Quality control is often performed by user acceptance testing. During User Acceptance Testing, Clients should identify how the results will be verified. Depending on the nature of the project, it is recommended that the Client develop a Testing Plan that includes Test Scripts, Testing Schedule, and Testing Signoff [6].

\subsubsection{Managing the SDLC (Systems Development Life Cycle)}

The Systems Development Life Cycle is the framework in which the actual development of the software or procedures occurs. The tasks on the project schedule should reflect the development, testing, and implementation during the Execution phase of the project. See the Appendix for more details.

\section{THE ROLE OF A PROJECT MANAGER IN AN AGILE PROJECT}

Agile projects not only follow a slightly different process than a tradition waterfall project, but some of the roles within the project team are slightly different as well. In some of the Agile methodologies, there is no specified "Project Manager" role. Instead, terms such as Scrum Master are used, as is the case in the Scrum Methodology. Team Lead is another term often used in Agile project team structures.

In agile projects, schedules (more specifically the content or deliverables of an increment or "sprint"), are more fluid. With the initiation of each new "sprint", the goal and expected output of the sprint is determined by analyzing a combination of the project's requirements, also referred to as a backlog, feedback from the customer regarding the current state of development, and any reprioritization of project objectives, needs, requirements, etc.[4]

While it's important to understand the objectives of the overall project, and to have a high-level idea in mind regarding the output of each sprint, project teams must be flexible and able to adjust to changing requirements, and/or re-prioritization of requirements.

In order to facilitate this approach, a person managing these processes must be flexible and ready to make adjustments quickly, rather than concentrate on adherence to a pre-defined plan. 


\section{SCRUM METHODOLOGY}

Scrum is an agile method for project management developed by Ken Schwaber[4]. It's goal is to dramatically improve productivity in teams previously paralysed by heavier, process-laden methodologies. Its intended use is for management of software development projects as well as a wrapper to other software development methodologies such as Extreme Programming.

Scrum is characterised by: A living backlog ofprioritised work to be done. Completion of a largely fixed set of backlog items in a series of short iterations or sprints. A brief daily meeting (called a scrum), at which progress is explained, upcoming work is described, and obstacles are raised. A brief planning session in which the backlog items for the sprint will be defined. A brief heartbeat retrospective, at which all team members reflect about the past sprint. Scrum is facilitated by a scrum master, whose primary job is to remove impediments to the ability of the team to deliver the sprint goal. The scrum master is not the leader of the team (as they are selforganising) but acts as a productivity buffer between the team and any destabilising influences.

Scrum enables the creation of self-organizing teams by encouraging verbal communication across all team members and across all disciplines that are involved in the project. A key principle of scrum is its recognition that fundamentally empirical challenges cannot be addressed successfully in a traditional "process control" manner. As such, scrum adopts an empirical approach - accepting that the problem cannot be fully understood or defined, focusing instead on maximising the team's ability to respond in an agile manner to emerging challenges.

\section{ORGANIZATION STANDARD METHOD OF PROJECTS AND MULTI-PROJECTS:}

Nowadays, waterfall model is considered a standard method for development process software.

In 1970, Winston W. Royce introduces method of dividing software development process into two stages for small projects, analysis and programming and into seven consecutive stages for big projects (Royce, 1970). This is a straight forward model, right now it is again transferred as waterfall model. Even if Royce's implicit repeat that was developing products with required complex plans, waterfall model is usually used to develop software has been formed for the year.

In 1990s, some style methods such as scrum have been planned as alternative for traditional driven approaches.A high modern framework for software engineering is called scrum 6 , 7, 8 against waterfall model that is in a so gradual and iterative approach during the software development. The general goal of the project is divided into sub-goals that have been added to total product.Role is scrum in classic hierarchy in the company. Shareholders are in head of the hierarchy. Product owner and product commission

$\mathrm{He}$ is responsible for product and connector between beneficiaries and product. On the next level sub-owner of product scrum master, a type of project manager and connecting link between product owner and real development team that has been determined scrum team. Depending on the project size to implement, several scrum masters and several development team can work on a project.Real development occurs during so called two speeds. These two speeds have been defined in a time framework in which requirements have been implemented by developers. After two speeds ended, the current situation has been evaluated and refinement in total product is possible. Two speeds again have been divided to daily scrum that during it briefly was discussed in the past day in where problems have occurred and solutions had been found was conducted. In addition, a plan for what should have been conducted in the day. This form of organization leading to parallel and transparency in working is possible due to frequent good rhythm meetings of evaluation.

Advantages of using the method are clear focus on solving certain tasks and as a result implementing the general solution and without high organizational cost. However, due to relatively unavailable communication and consulting between developers, high losing of economic welfare exists. Major problems of this organization evaluation development model and lost coordinating among sub-team. In the second mode, significantly intensifying development of sub-project is with powerful dependence. In addition, there is an evaluation of results that usually has been conducted by self-developers.

Agility is higher than flexibility and adaptability. Agility is modifiability and adaptability in a turbulent environment which is with intelligence, creativity and innovation. Some qualitative attributes have been briefly explained in table 1 .

Table 1. Explaining some qualitative attributes [12].

\begin{tabular}{|l|l|}
\hline Agility & $\begin{array}{l}\text { Agility: modifiabilityand adaptability in a turbulent environment }+ \text { intelligence, creativity and } \\
\text { innovation }\end{array}$ \\
\hline $\begin{array}{l}\text { Modifiability } \\
\text { or } \\
\text { Changeable }\end{array}$ & $\begin{array}{l}\text { Modifiability that includes portability and reusability and some other features. } \\
\text { It is a modifiable or changeable if the least possible number of its separate elements are involved in } \\
\text { process of changes. A changeable system has a wide concept and using that about a system has some } \\
\text { ambiguities. }\end{array}$ \\
\hline Adaptability & $\begin{array}{l}\text { Adaptability is a qualitative features of software that some systems need it due to special conditions } \\
\text { that environment force to them. Adaptability is a special type of flexibility and it is raised when a } \\
\text { system with a high frequency requires changes. An adaptable system needs a adaptable architecture. } \\
\text { Adaptability about behavioral aspects is harder than adaptability about structural aspects. Behavioral } \\
\text { aspects are too complex and those actions which will be needed in future are not predictable. }\end{array}$ \\
\hline Flexibility & Flexibility is responsiveness to specified changes. Root of many problems in a turbulent organization is \\
\hline
\end{tabular}


So comparing agile with available methodologies is not a useful work because they are neither aligned nor family. Even rup can be used within agile but not their rup but our custommade rup (organization). So instead of comparing we should think about how can we become agile [9][10].

\section{CONCLUSION}

Although agility project management is a daunting task, organizations that implement effective processes proved to be successful, while those that fail in this effort will be unsuccessful. The nature of software projects creates many risks that must be managed diligently to avoid the common drawback of many projects. The perceptions and attitudes towards risk management activities compound difficult challenges for implementing a risk management strategy.Formal risk management process is recommended to manage complex issues associated with software development projects.

Many risk management processes have been created to aid organizations, but integrating the processes into organizations was not successful. The theoretical aspects of the process must be reconciled with the practical challenges of the organization to implement risk management successfully. Effective risk management process will succeed by changing the organizational culture to motivate the individual. Cultural changes require time and repetition before they are firmly embedded into the organization.

Agile teams have confirmed for faster increase in $37 \%$ profit and producing income 30 percent more than non-agile companies, higher speed, flexibility and productivity have been obtained. However, always there is a place for variety. Depending on our goals and business needs, we may still benefit waterfall model or a combination of both of them. Goal of this article is finding a suitable method for project teams. Rugby is a simple game and has simple rules for learning and it is accurate and regular.Traditional modeling methods are based on documentations and stationary reports which are not responsible for increasing changes of users' needs in environments that are changing rapidly.Pre- development heavy designing and primary comprehensive and major planning in traditional methods made experts to think about new methods to develop software. A solution to solve these problems is using agile modelings that are specified by two indexes of adaptability and flexibility. Unlike traditional methods that believe in existence of solution for each problem. In agile method, idea is that tailored to needs and tastes of each person a different solution can be provided to solve the problem. In this article, some challenges of traditional methods are evaluated and existing problems are explained. In following, some methods for agile development and then advantages of using these methods are explained.

\section{REFERENCES}

[1] Augustine, S. Managing agile projects: Prentice Hall PTR.Begel, A., \& Nagappan, N. Usage and perceptions of agile software developmentin an industrial context: An exploratory study. In Empirical Software Engineering and Measurement, vol2 , no3 2014

[2] https://www.versionone.com/pdf/state-of-agiledevelopment-survey-ninth.pdf

[3] M. Fowler, J. Highsmith, The agile manifesto, Software Development vol .3, no 2 p28-35, 2015

[4] https://www.scrumalliance.org/scrum

[5] Beck K, Extreme Programming Explained-Embrace Change, Addison-Wesley, 2015.

[6] K. Schwaber, M. Beedle, Agile Software Development with Scrum, Prentice-Hall,2014.

[7] Cockburn, Agile Software Development, AddisonWesley, 2012.

[8] S.M. Palmer, J.M. Felsing, A Practical Guide to FeatureDriven Development, Prentice-Hall, USA, 2015.

[9] Len Bass, Paul Clements, Rick Kazman "Software Architecture inPractice, Second Edition"vol 4, 2015

[10] H. Kashanian, M. Peashdad, and M. Kondori, "Development of umbrella activities in agile methodologies," Int. Acad. J. Innov. Res., vol. 3, no. 1, pp. 27-31, 2016. 